"This is the peer reviewed version of the following article: Xiao L, Alder R, Mehta M, Krayem N, Cavasinni B, Laracy S, Cameron S, Fu S, (2017) Development of a quantitative method for the analysis of cocaine analogue impregnated into textiles by Raman spectroscopy, Drug testing and analysis, 10(4):761-767. which has been published in final form at https://doi.org/10.1002/dta.2261. This article may be used for non-commercial purposes in accordance with Wiley Terms and Conditions for SelfArchiving." 


\title{
Development of a quantitative method for the analysis of cocaine analogue impregnated into textiles by Raman spectroscopy
}

Linda Xiao, ${ }^{\mathrm{a}}$ Rhiannon Alder, ${ }^{\mathrm{a}}$ Megha Mehta, ${ }^{\mathrm{a}}$ Nadine Krayem, ${ }^{\mathrm{a}}$ Bianca Cavasinni, ${ }^{\mathrm{a}}$ Sean Laracy, Shane Cameron ${ }^{\mathrm{b}}$ and Shanlin $\mathrm{Fu}^{\mathrm{a} *}$

${ }^{a}$ Centre for Forensic Science, University of Technology Sydney, PO Box 123, Broadway, NSW 2007, Australia

b Specialist Operations, Australian Federal Police, 110 Goulburn St, Sydney, NSW 2000, Australia

* Correspondence to: Shanlin Fu, Centre for Forensic Science, University of Technology Sydney, PO Box 123, Broadway, NSW 2007, Australia.

E-mail: shanlin.fu@uts.edu.au

Short title: Raman analysis of cocaine anologue impregnated into textiles

\begin{abstract}
Cocaine trafficking in the form of textile impregnation is routinely encountered as a concealment method. Raman spectroscopy has been a popular and successful testing method used for in situ screening of cocaine in textiles and other matrices. Quantitative analysis of cocaine in these matrices using Raman spectroscopy has not been reported to date. This study aimed to develop a simple Raman method for quantifying cocaine using atropine as the model analogue in various types of textiles. Textiles were impregnated with solutions of atropine in methanol. The impregnated atropine was extracted using less hazardous acidified water with the addition of potassium thiocyanate (KSCN) as an internal standard for Raman analysis. Despite the presence of background matrix signals arising from the textiles, the cocaine analogue could easily be identified by its characteristic Raman bands. The successful use of KSCN normalised the analyte signal response due to different textile matrix background interferences and thus removed the need for a matrix-matched calibration. The method was linear over a concentration range of $6.25-37.5 \mathrm{mg} / \mathrm{cm}^{2}$ with a coefficient of determination $\left(\mathrm{R}^{2}\right)$ at 0.975 and acceptable precision and accuracy. A simple and accurate Raman spectroscopy method for the analysis and quantification of a cocaine analogue impregnated in textiles has been developed and validated for the first time. This proof-of-concept study has demonstrated that atropine can act as an ideal model compound to study the problem of cocaine impregnation in textile. The method has the potential to be further developed and implemented in real world forensic cases.
\end{abstract}

Keywords: Cocaine; Atropine, Drug trafficking; Textile Impregnation; Raman Spectroscopy 


\section{INTRODUCTION}

Authorities face many issues when combating the problem of the illicit drug market. The main two issues are the constant emergence of new drug analogues and the new techniques smugglers use for the transportation of illicit drugs. Many methods of drug concealment have been reported, ranging from the traditional body packing to the more creative methods of polymer impregnation, ${ }^{[1]}$ concealment within book bindings ${ }^{[2]}$ and wax blocks, ${ }^{[3]}$ suspensions in liquids including milk ${ }^{[4-6]}$ and impregnation in clothing. ${ }^{[4,7-9]}$

Screening techniques, such as those used by Customs and Border Protection, for qualitative detection of drugs include colour tests, ${ }^{[5]}$ ion mobility spectroscopy ${ }^{[5]}$ and portable Raman and Fourier transform infrared (FTIR) spectroscopy. ${ }^{[8]}$ Raman and FTIR are also considered as Category A analytical techniques according to the SWGDRUG guideline ${ }^{[10]}$ with high discrimating power when used for drug identification and confirmation.

Raman spectroscopy has gained rapid development in the last decade to be used as a valuable forensic tool for the analysis of drugs of abuse including cocaine in different types of forensic evidence. ${ }^{[11]}$ It allows for non-destructive, portable and in situ analysis of cocaine in many matrices, including tablets or powders, some solvents, ${ }^{[5,12-15]}$ and to a lesser extent textiles. ${ }^{[9]}$ The quantitative analysis of cocaine impregnated in textiles by using Raman has not been reported in the literature.

The aim of this proof-of-concept study was to develop and validate a method suitable for the detection and quantification of a cocaine analogue, atropine sulfate monohydrate, in various textiles by using Raman spectroscopy. The developed method required the use of internal standard potassium thiocyanate $(\mathrm{KSCN})$ and a simple extraction of the drug analogue from the matrix into acidified water. Validation studies were performed on specific parameters including linearity, precision, accuracy, limit of detection (LOD), and limit of quantification (LOQ). The method was also evaluated using cocaine to show the similarity of results between drug analogue and drug.

\section{MATERIALS AND METHODS}

\section{Chemicals}

Solid analytical reagent grade (AR) atropine sulfate monohydrate and AR 98\% sulfuric acid $\left(\mathrm{H}_{2} \mathrm{SO}_{4}\right)$ were purchased from Sigma-Aldrich (Sydney, NSW, Australia). Cocaine hydrochloride (Cocaine.HCl) was obtained from the National Measurement Institute (North Ryde, NSW, Australia). Solid AR KSCN from BDH (Tingalpa, QLD, Australia) was used as internal standard. The impregnation solvent AR methanol was obtained from Chem-Supply (Gillman, SA, Australia).

Stock solutions were prepared at concentrations of $20 \%(\mathrm{w} / \mathrm{v})$ atropine in $0.5 \mathrm{M} \mathrm{H}_{2} \mathrm{SO}_{4}, 20 \%(\mathrm{w} / \mathrm{v}$ ) atropine in methanol, $10 \%(\mathrm{w} / \mathrm{v}) \mathrm{KSCN}$ in $\mathrm{H}_{2} \mathrm{O}, 20 \%(\mathrm{w} / \mathrm{v})$ cocaine in $0.5 \mathrm{M} \mathrm{H}_{2} \mathrm{SO}_{4}$ and $20 \%(\mathrm{w} / \mathrm{v})$ cocaine in methanol.

\section{Textiles impregnation and extraction}

A selected set of fibres were used in this study to represent a range of textile materials, these included natural and synthetic textiles. Wool and cotton were used as natural fibres. Polyester fibres were used to represent synthetic fibres. The textiles used were a white towel (cotton), blue denim jeans (cotton), red singlet (polyester), purple jumper (polyester/wool), check shirt (cotton/polyester), and black jumper (wool), as shown in Figure 1. Textile pieces $(2 \mathrm{~cm} \times 2 \mathrm{~cm})$ were impregnated with $20 \%(\mathrm{w} / \mathrm{v})$ atropine in methanol. The textile swatches were hung with clips and 
impregnated by slowly pipetting the solution on. Care was taken so that the textiles were not saturated with the solution at any given time. After overnight drying, swatches were placed in a plastic vial, where $250 \mu \mathrm{L}$ of the internal standard $(10 \% \mathrm{KSCN}$ in water, w/v) was added and the solution was made up to $1 \mathrm{~mL}$ using $0.5 \mathrm{M} \mathrm{H}_{2} \mathrm{SO}_{4}$. The tubes used for these samples were agitated for 2 minutes and the aqueous solution was used for Raman analysis.

[insert Figure 1 here]

\section{Raman spectroscopy}

All samples were analysed using a Renishaw inVia Raman microscope and spectra analysed with the Renishaw WiRE 3.4 software. The source laser was $633 \mathrm{~nm}$ over the range of $120-4000 \mathrm{~cm}^{-1}$. Spectra were collected with 20x magnification, 100\% laser power, 10s exposure time and 4 accumulations.

\section{Quantitative analysis and method validation}

Target peaks, at $1003 \pm 1 \mathrm{~cm}^{-1}$ for both atropine and cocaine and $2067 \pm 1 \mathrm{~cm}^{-1}$ for the internal standard KSCN, from the Raman spectra produced were used for the quantitative analysis. In order to obtain the peak ratios used for quantitative analysis, the target peak area of the analyte was divided by the target peak area of the internal standard.

Method validation was in accordance with published procedures ${ }^{[16,17]}$ and fit for purpose. Denim textile was used for method validation due to its reported popularity in drug impregnation and trafficking. ${ }^{[9]}$ Linearity was investigated in the range of 2.5 to $50 \mathrm{mg} / \mathrm{cm}^{2}$ impregnated atropine in triplicate and determined through the construction of calibration curves and using a line of best fit. The LOD and LOQ were the lowest concentrations with the signal-to-noise $(\mathrm{S} / \mathrm{N})$ ratio of 3 and 10 , respectively. For LOQ, the calculated concentration should also be within $20 \%$ relative error (RE) of the expected concentration. The precison and accuracy were determined using quality control samples impregnated at $10.00 \mathrm{mg} / \mathrm{cm}^{2}$ (low QC) and $20.00 \mathrm{mg} / \mathrm{cm}^{2}$ (high QC), respectively ( $\mathrm{n}=3$ ). The intra-day study was done in triplicates and the inter-day study was carried out for three consecutive days. If the calculated concentrations fell within $\pm 20 \%$ of known spiked concentration (as represented by percentage mean relative error or MRE), the method was considered accurate. Precision was calculated using percentage relative standard deviation (RSD) and was deemed acceptable if it was $<15 \%$.

\section{RESULT AND DISCUSSION}

\section{Use of atropine as the substitute for cocaine impregnation into textiles}

Impregnation of cocaine into textiles according the current study protocol requires a substantial amount of cocaine material. Cocaine is a controlled substance in Australia, and its possession and the amount one can possess for scientific research purpose are subject to regulatory control. Due to the close structural similarity and ease of acquisition, atropine was used as the substitute for cocaine for proof-of-concept investigation in this study.

The Raman spectra of pure solid atropine sulfate monohydrate and cocaine hydrochloride are given in Figure 2. Cocaine could be identified by the three major peaks at 1000,1598 and $1715 \mathrm{~cm}^{-1}$, in agreement with those reported by Weyermann and co-authors. ${ }^{[5]}$ Although the two spectra have many similar peaks due to their structural similarities, there are two main shifts which distinguish the two drugs. One of these peaks is the $751 \mathrm{~cm}^{-1}$ peak which is prominent in the atropine spectrum 
but almost not visible for the cocaine spectrum. The other major difference is the $\mathrm{C}=\mathrm{O}$ ester peaks which were present at $1729 \mathrm{~cm}^{-1}$ for atropine and $1715 \mathrm{~cm}^{-1}$ for cocaine.

[insert Figure 2 here]

\section{Impregnation of drug analyte into textiles}

Methanol was used as the impregnation solvent due to its fast drying nature when compared with using acidified water (data not shown). Direct Raman spectroscopic analysis was performed on atropine-impregnated denim at both $10.00 \mathrm{mg} / \mathrm{cm}^{2}$ and $18.75 \mathrm{mg} / \mathrm{cm}^{2}$ concentrations. Prior to Raman analysis, microscopic examination of the denim samples was performed on the Leica application suite software at 20x magnification, as shown in Figure 3. At $18.75 \mathrm{mg} / \mathrm{cm}^{2}$ there was a clear and relatively even distribution of atropine found imbedded into the textile fibres. Hence, a suitable spectrum was acquired. At $10.00 \mathrm{mg} / \mathrm{cm}^{2}$, due to inhomogeneity of drug absorption, usable spectra could not be obtained unless the microscope was properly focused on drug crystals on the fibres.

[insert Figure 3 here]

The visible drug analogue on the clothing under optical examination correlates to the case reported by McDermott and Power, ${ }^{[9]}$ in which 6 seized garments were found to contain cocaine soaked into the denim fabric. The visible starch-like appearance of both textiles suggests that the concentrations used in this study may well represent the concentrations that have been used to smuggle drugs in the real world.

\section{Extraction of drug analyte from the impregnated matrix}

Two extraction methods were tested, these being liquid-liquid extraction (LLE) using water and dichloromethane and a straight extraction using $0.5 \mathrm{M} \mathrm{H}_{2} \mathrm{SO}_{4}$. The LLE method was not used for the final analysis as it extracted some of the dyes and caused fluorescence in the Raman spectra (data not shown). The $0.5 \mathrm{M} \mathrm{H}_{2} \mathrm{SO}_{4}$ solution resulted in the least dye extracted and, therefore, the cleanest Raman spectrum. The other advantage to this is that the low concentration acid is less hazardous to use compared to strong organic solvents. This would be particularly beneficial if the technique was to be implemented in regular drug testing analysis, especially when a large volume of extraction solvent is required to handle a bulky textile item such as a whole pair of denim jeans or a garment.

In addition to the black woolen jumper, a number of other dark coloured textiles such as a dark green polyester/coton shirt were also investigated in the study. It was found that significant amounts of dyes were extracted into the extraction solvent and produced strong fluorescence which masked the atropine Raman signals (data not shown). The issue of dye fluorescence was also encountered by Ali et al. ${ }^{[4]}$ when analysing an orange textile impregnated with cocaine hydrochloride. The interference of fluorescence from dark dyes may not be a real world limitation of the method, because a drug smuggler may be less likely use dark coloured textiles for impregnation as cocaine forms a white powder precipitate and would be visible without the need for complex analysis. Due to this, the dark textiles were not exhaustively investigated in the study.

\section{Use of internal standard KSCN}

To investigate the effect of different textiles on the Raman signal response, three swatches of representative textiles were impregnated with $18.75 \mathrm{mg} / \mathrm{cm}^{2}$ atropine in methanol and then subjected to solvent extraction and Raman analysis $(n=3)$. The results were summarised in Figure 4. 
[insert Figure 4 here]

Without correction using the KSCN peak, the target peak area of the atropine varied significantly across the different materials and there was a large error in the measurement. The blue denim jeans experienced the largest variation in terms of the absolute peak area of atropine, with a 65\% RSD. This result was probably due to the uneven distribution of the dye on the denim fabric. The ratio of the atropine peak area to the KSCN peak area standardised the measurement across the various textiles and reduced the error. KSCN was therefore considered a suitable internal standard for the study. The use of the internal standard can remove the need to perform matrix-matched calibration, providing a 'one size fits all' calibration curve for drug analysis to be performed in different types of textile under investigation.

\section{Linearity}

Triplicate samples were prepared using denim textile for each concentration to evaluate the linearity for statistical purposes. Average peak area ratios of atropine to internal standard peak were used for sample quantification through these calibration curves. The linearity of the Raman method was found within the range $6.25-37.50 \mathrm{mg} / \mathrm{cm}^{2}$. The coefficient of determination values $\left(\mathrm{R}^{2}\right)$ of the calibration curves were greater than or equal to 0.975 . The calibration curve for solutions of atropine sulfate in acidified water extracted from the blue denim is shown in Figure 5.

[insert Figure 5 here]

\section{Limit of detection (LOD) and limit of quantification (LOQ)}

The lowest amount of an analyte in a sample which could be detected, but not necessarily quantitated, was estimated as $3.13 \mathrm{mg} / \mathrm{cm}^{2}$ (LOD) and the lowest amount of an analyte in sample which was able to be quantitatively determined was $6.25 \mathrm{mg} / \mathrm{cm}^{2}$ (LOQ).

\section{Precision and accuracy}

Variations of results within the same day (intra-day) and between days (inter-day) were analysed and summarised in Table 1. The method was found to be reproducible and accurate when atropine was impregnated in denim. Both precision and accuracy values are excellent and were well within the recommended values of $\mathrm{RSD}<15 \%$ and MRE $< \pm 20 \%$, respectively.

[insert Table 1 here]

\section{Comparison of Raman spectra of cocaine and atropine in the extraction solvent}

This study was a proof-of-concept to determine whether atropine could be utilised as a model compound to develop methods for the analysis and quantification of cocaine impregnated in textile. It was not feasible to use cocaine in the impregnation study as it is expensive and the substantial amounts required do not comply to regulations. In order for atropine to be used as a model the peaks and target peak to internal standard peak ratio must be similar between atropine and cocaine. The spectra from 5\% solutions of atropine and cocaine with $2.5 \%$ internal standard in the extraction solvent $0.5 \mathrm{M} \mathrm{H}_{2} \mathrm{SO}_{4}$ are given in Figure 6.

[insert Figure 6 here]

As seen previously in the spectra of the solid samples, atropine and cocaine in solution have very 
similar peaks, the most notable being $981 \mathrm{~cm}^{-1}, 1003 \pm 1 \mathrm{~cm}^{-1}, 1052 \mathrm{~cm}^{-1}$ and $1603 \mathrm{~cm}^{-1}$. The main differences between the spectra for the solids and the solutions are the less defined baselines and the additional peak at $2067 \pm 1 \mathrm{~cm}^{-1}$ which corresponds to the addition of internal standard, KSCN. The similarity in the peaks allowed for the peak at $1003 \pm 1 \mathrm{~cm}^{-1}$ to be used as the target peak for the quantification analysis of both compounds.

The procedure was repeated to analyse standard solutions for both cocaine and atropine with concentrations of $2.5 \%, 4 \%, 5 \%, 7.5 \%$ and $10 \%$ in $0.5 \mathrm{M} \mathrm{H}_{2} \mathrm{SO}_{4}$ with $2.5 \% \mathrm{KSCN}$ solution. The concentration range correlated to the concentration range investigated during the impregation study described earlier. In order to determine the linearity, the peak area for the target peak of the analyte of interest was divided by the peak area of the internal standard peak. This ratio was plotted against the known concentration to form the calibration curves. A linear trend was observed for the calibration curves of both cocaine and atropine with the $\mathrm{R}^{2}$ close to 1, as shown in Figure 7.

[insert Figure 7 here]

Using the atropine calibration curve, a $6.05 \%$ cocaine solution gave a $6.25 \%$ calculated cocaine concentration, yielding a $+3.3 \%$ concentration error. This shows the similarity of the response between atropine and cocaine at $1003 \mathrm{~cm}^{-1}$ when standardised by the internal standard. Therefore, atropine is considered a good model compound for developing Raman-based testing methods to detect and quantify cocaine impregnated in textile.

\section{CONCLUSION}

A simple and accurate Raman spectroscopy method for the analysis and quantification of cocaine analogue impregnated in textile has been developed and validated for the first time. The significant advantage of the method is the addition of the KSCN internal standard offering superb repeatability and accuracy. The successful utilisation of the internal standard removes the need to construct matrix-matched calibrations. The availability of this 'one size fits all' calibration curve allows for drug analysis to be performed in different types of textile under investigation. The use of dilute acidic water instead of the conventional organic solvents for extraction offers a more user friendly alternative with reduced health risk to both humans and the environment. This proof-of-concept study has demonstrated that atropine can act as an ideal model compound to study the problem of cocaine impregnation in textile. Despite the good correlation observed between cocaine and atropine in terms of their Raman responses and concentration calculation, it is advisable that the impregation study be repeated with cocaine when conditions permit and the method be further validated using real forensic samples i.e. seized textile items impregnated with cocaine when available. Nevetheless the study represents a significant contribution to the field of drugs of abuse testing and paves the way for future development, advancement and implementation of sensitive and accurate methods for testing cocaine in non-conventional matrices by using Raman spectroscopy. 


\section{REFERENCES}

[1]. U.S. Department of Justice Drug Enforcement Administration. Cocaine in a hard rubber-like matrix in Tulua, Colombia. Microgram Bull 2007; 40(5): 51.

[2]. U.S. Department of Justice Drug Enforcement Administration. Hard cover books containing cocaine in New York. Microgram Bull 2004; 37(4): 66.

[3]. U.S. Department of Justice Drug Enforcement Administration. Cocaine in a very large block of wax in New York. Microgram Bull 2007; 40(1): 2.

[4]. Ali EMA, Edwards HGM, Hargreaves MD, Scowen IJ. In situ detection of cocaine hydrochloride in clothing impregnated with the drug using benchtop and portable Raman spectroscopy. J Raman Spectrosc 2010; 41(9): 938-943.

[5]. Weyermann C, Mimoune Y, Anglada F, et al. Applications of a transportable Raman spectrometer for the in situ detection of controlled substances at border controls. Forensic Sci Int 2011; 209(1-3): 21-28.

[6]. Eliasson C, Macleod NA, Matousek P. Non-invasive detection of cocaine dissolved in beverages using displaced Raman spectroscopy. Anal Chim Acta 2008; 607(1): 50-53.

[7]. Ali EMA, Edwards HGM, Scowen IJ. Rapid in situ detection of street samples of drugs of abuse on textile substrates using microRaman spectroscopy. Spectrochim Acta A 2011; 80(1): 2-7.

[8]. Ali EMA, Edwards HGM. Screening of textiles for contraband drugs using portable Raman spectroscopy and chemometrics. J Raman Spectrosc 2014; 45(3): 253-258.

[9]. McDermott S, Power J. Drug Smuggling Using Clothing Impregnated with Cocaine. $J$ Forensic Sci 2005; 50(6): 1423-1425.

[10]. SWGDRUG SWGftAoSD. Scientific Working Group for the Analysis of Seized Drugs (SWGDRUG) recommendations http://swgdrug.org/Documents/SWGDRUG Recommendations Version 7-1.pdf. 2016: accessed 20 June 2017.

[11]. West MJ, Went MJ. Detection of drugs of abuse by Raman spectroscopy. Drug Test Anal 2011; 3: 532-538.

[12]. West MJ, Went MJ. The spectroscopic detection of drugs of abuse in fingerprints after development with powders and recovery with adhesive lifters. Spectrochim Acta A 2009; 71(5): 1984-1988.

[13]. West MJ, Went MJ. The spectroscopic detection of drugs of abuse on textile fibres after recovery with adhesive lifters. Forensic Sci Int 2009; 189(1-3): 100-103.

[14]. Eliasson C, Macleod NA, Matousek P. Non-invasive detection of powders concealed within diffusely scattering plastic containers. Vib Spectrosc 2008; 48(1): 8-11.

[15]. Moreno VM, López-López M, Atoche J-C, García-Ruiz C. Raman identification of drug of abuse particles collected with colored and transparent tapes. Sci Justice 2014; 54(2): 164169.

[16]. Molnar A, Lewis J, Doble P, et al. A rapid and sensitive method for the identification of delta-9-tetrahydrocannabinol in oral fluid by liquid chromatography-tandem mass spectrometry. Forensic Sci Int 2012; 215(1-3): 92-96.

[17]. Peters FT, Drummer OH, Musshoff F. Validation of new methods. Forensic Sci Int 2007; 165(2): 216-224. 
Table 1. Summary of method validation results including linearity, LOD, LOQ, precision and accuracy measurements

\begin{tabular}{|c|c|c|}
\hline & Acceptance Criteria & Results \\
\hline Linearity Range & & $6.25-37.50 \mathrm{mg} / \mathrm{cm}^{2}$ \\
\hline $\mathrm{R}^{2}$ & $>0.95$ & 0.975 \\
\hline LOD & $\mathrm{S} / \mathrm{N}>3: 1$ & $3.13 \mathrm{mg} / \mathrm{cm}^{2}$ \\
\hline LOQ & $\mathrm{S} / \mathrm{N}>10: 1 ; \% \mathrm{RE}< \pm 20 \%$ & $6.25 \mathrm{mg} / \mathrm{cm}^{2}$ \\
\hline Precision (\%RSD) & $\begin{array}{r}\text { low QC } \\
\text { high QC }\end{array}$ & $\begin{array}{ll}\text { Intra-day } & \text { Inter-day } \\
1.98 \% & 1.91 \% \\
1.56 \% & 3.17 \%\end{array}$ \\
\hline Accuracy (\%MRE) & $\begin{array}{r}\text { low QC } \\
\text { high QC }\end{array}$ & $\begin{array}{ll}\text { Intra-day } & \text { Inter-day } \\
0.94 \% & 1.23 \% \\
1.25 \% & 1.65 \%\end{array}$ \\
\hline
\end{tabular}




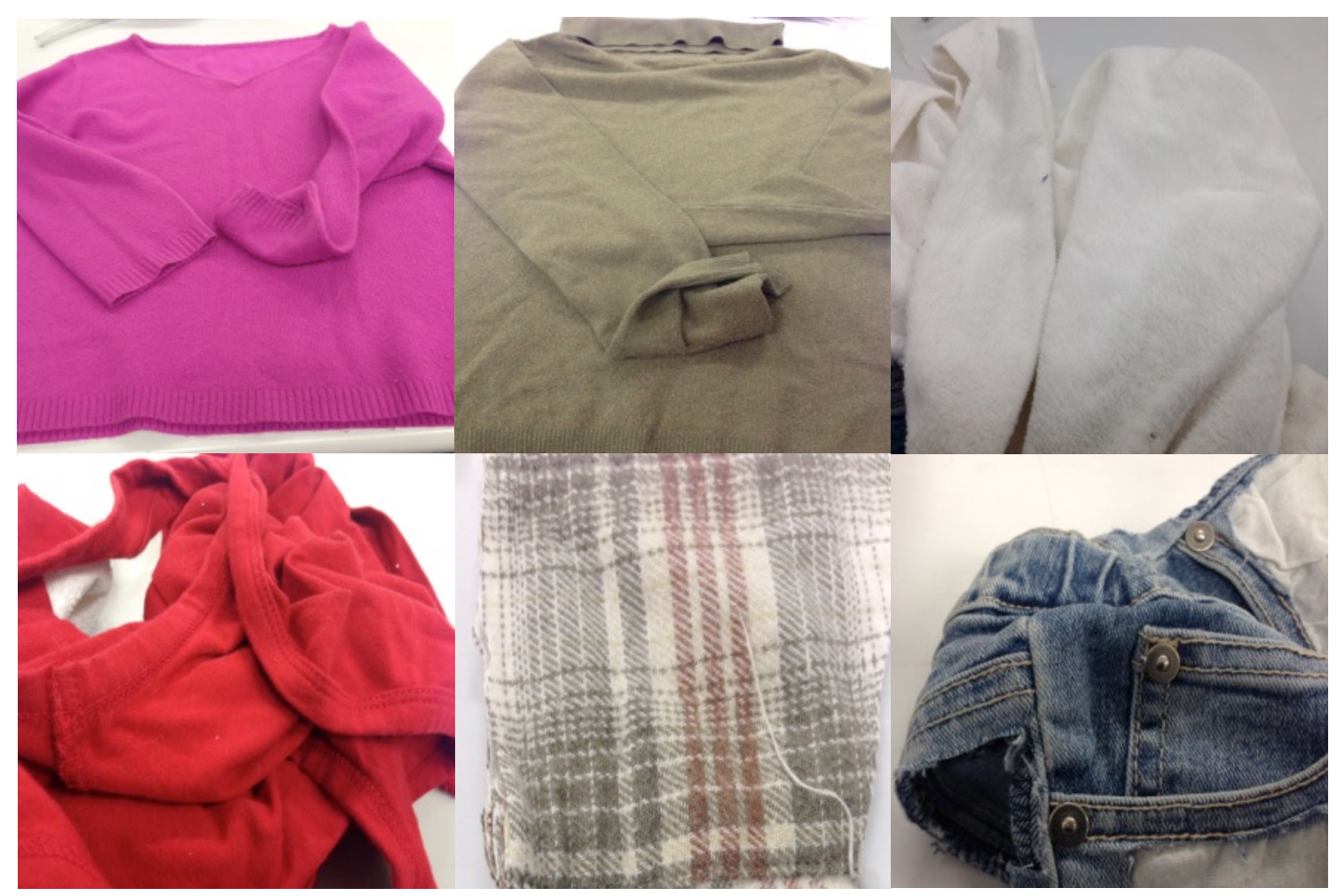

Figure 1. From top left (clockwise), purple jumper (polyester/wool), brown sweater (wool), white towel (cotton), blue denim jeans (cotton), check shirt (cotton/polyester), and red singlet (polyester). 

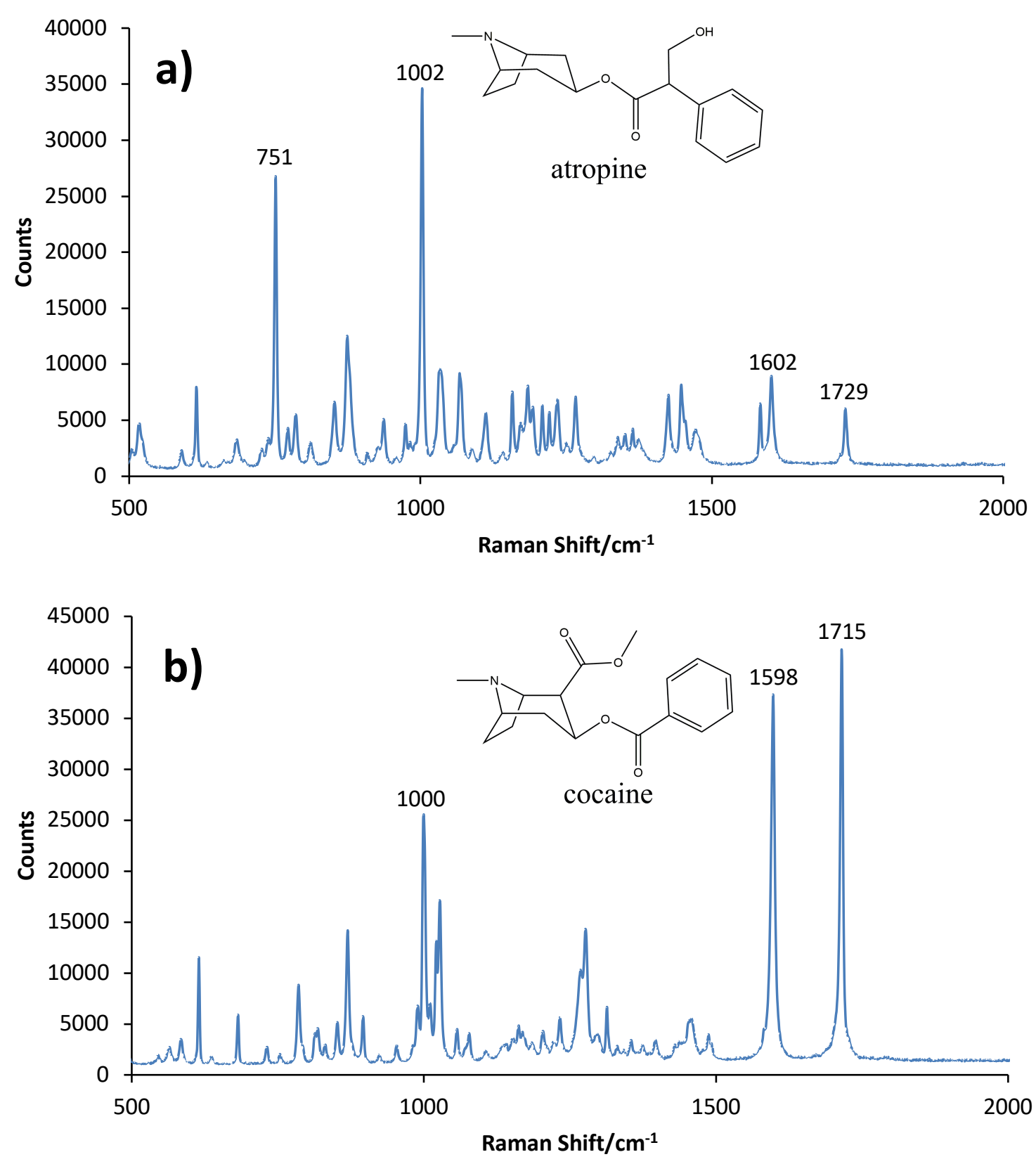

Figure 2. Raman spectra of solid atropine sulfate (a) and solid cocaine hydrochloride (b). 


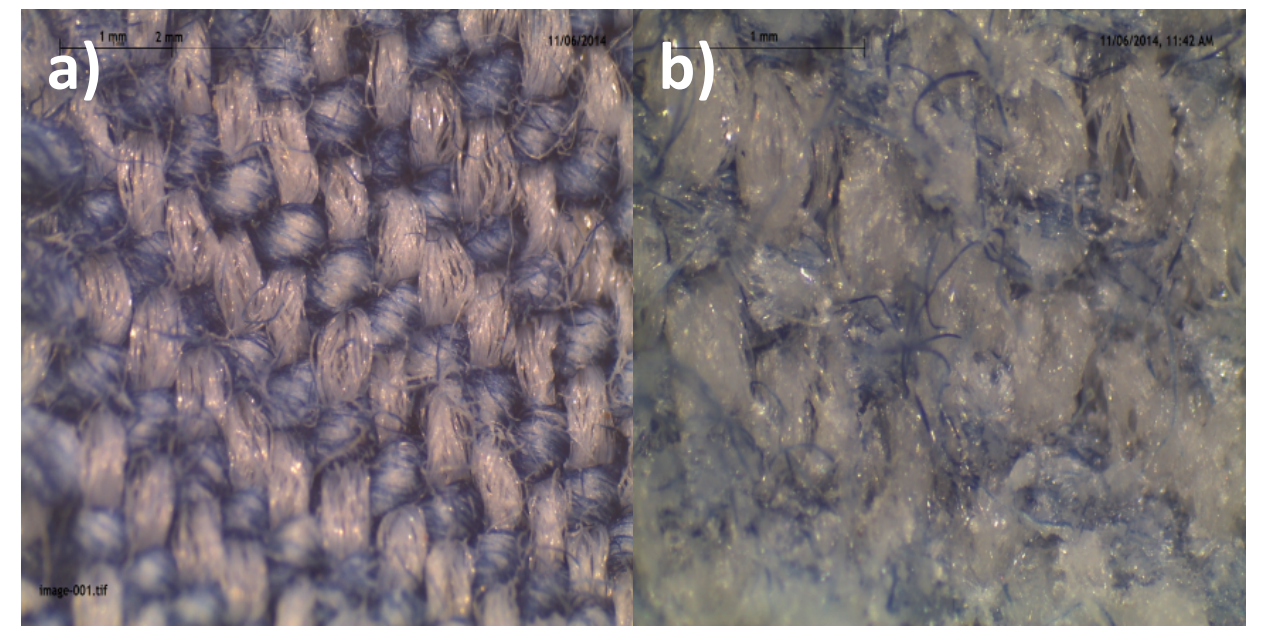

Figure 3. Microscopic images of $10.00 \mathrm{mg} / \mathrm{cm}^{2}$ (a) and $18.75 \mathrm{mg} / \mathrm{cm}^{2}$ (b) atropine impregnated in blue denim using Leica EZ4 D stereomicroscope with 10x eyepieces. 


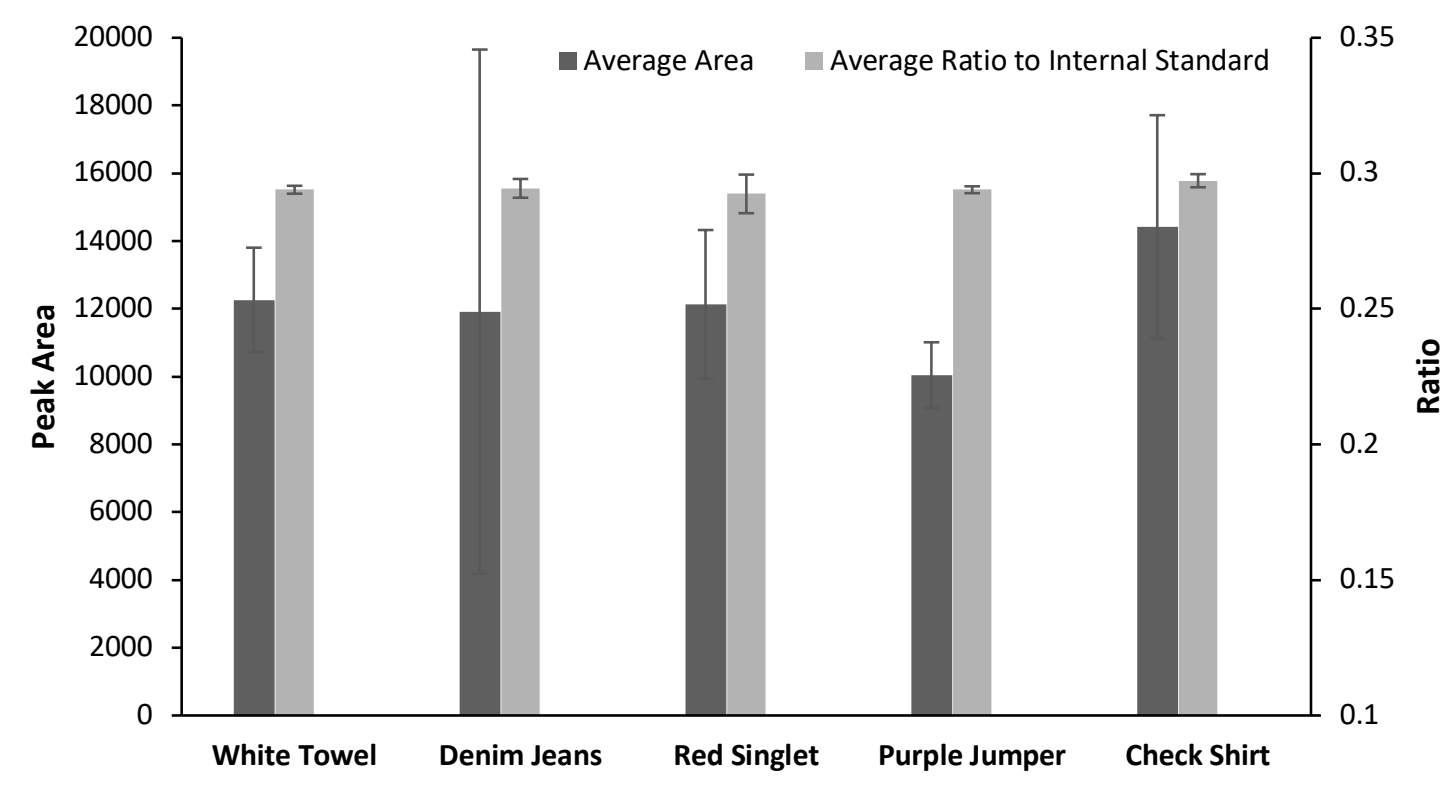

\begin{tabular}{|l|l|l|l|l|l|}
\cline { 2 - 6 } & While Towel & Denim Jeans & Red Singlet & Purple Jumper & Check Shirt \\
\cline { 2 - 6 } Peak Area & 12,300 & 11,900 & 12,100 & 10,000 & 14,400 \\
\cline { 2 - 6 } RSD (Peak Area) & $13 \%$ & $65 \%$ & $18 \%$ & $10 \%$ & $23 \%$ \\
Ratio & 0.29 & 0.30 & 0.29 & 0.29 & 0.30 \\
\cline { 2 - 6 } RSD (Ratio) & $0.5 \%$ & $1.2 \%$ & $2.4 \%$ & $0.4 \%$ & $0.8 \%$ \\
\cline { 2 - 6 } & & \multicolumn{2}{|l}{} & &
\end{tabular}

Figure 4. Raman signal response of $18.75 \mathrm{mg} / \mathrm{cm}^{2}$ atropine extracted from different textiles, expressed as the absolute peak area or the ratio relative to the internal standard KSCN. Data was collected by means of triplicate measurements with the standard deviation represented by the error bars. 


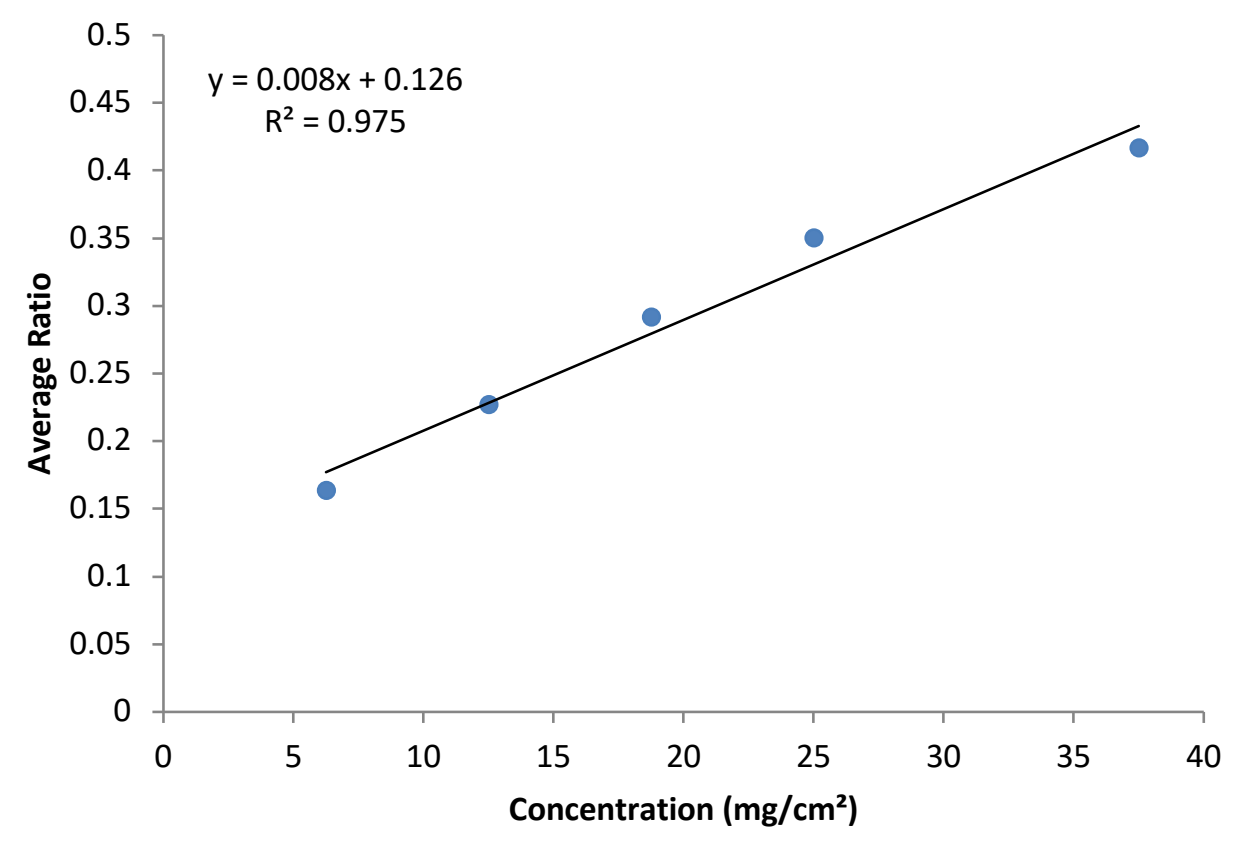

Figure 5. Calibration curve for solutions of atropine in acidified water extracted from blue denim. 

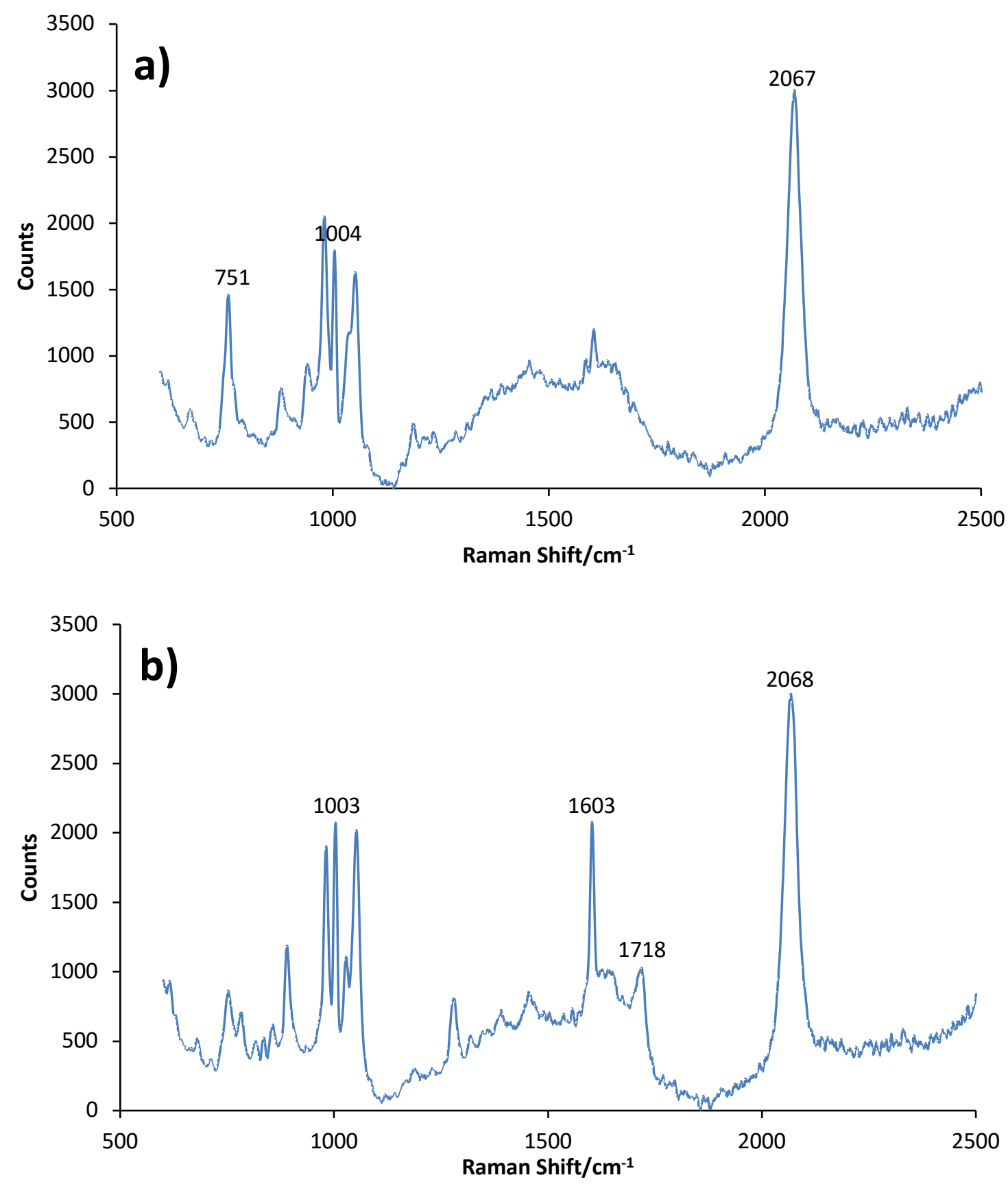

Figure 6. Raman spectra of 5.0\% atropine (a) and 5.0\% cocaine (b) in $0.5 \mathrm{M} \mathrm{H}_{2} \mathrm{SO}_{4}$ with $2.5 \%$ KSCN. 

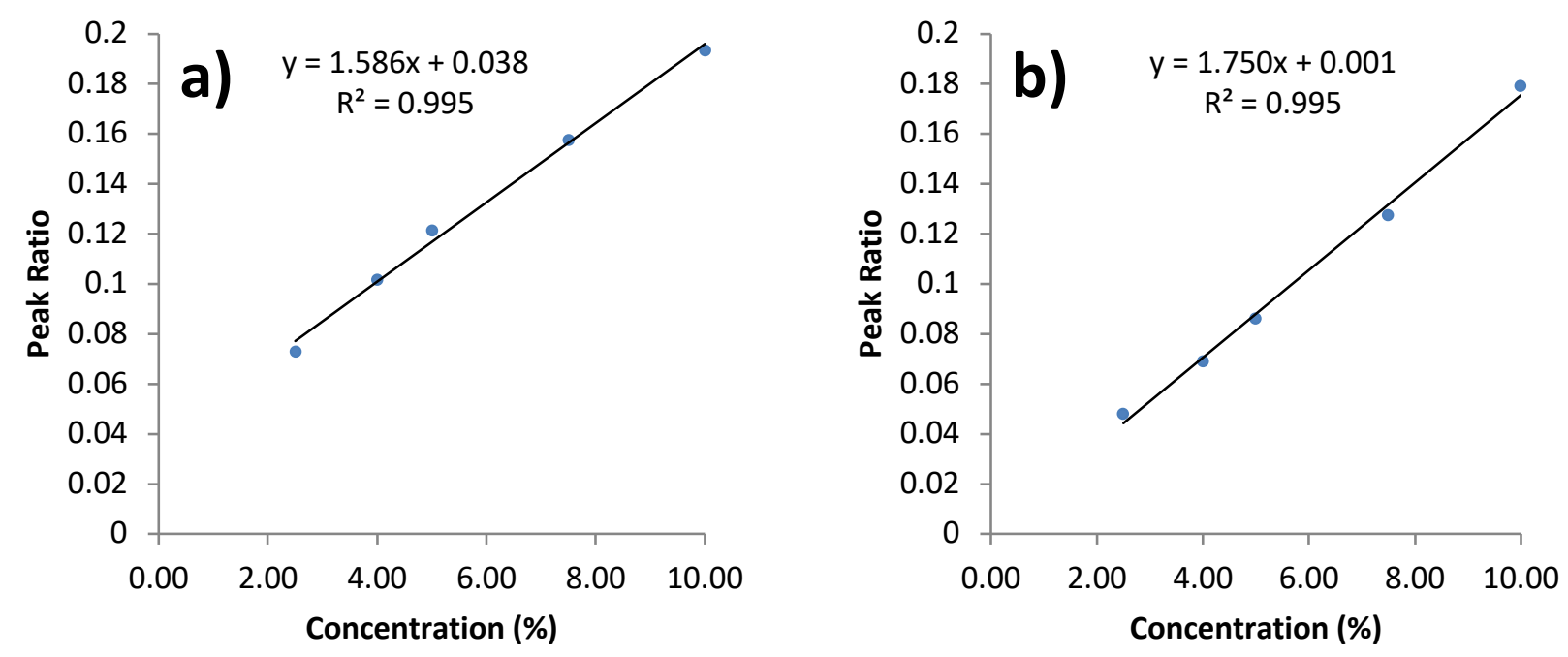

Figure 7. Calibration curves for cocaine (a) and atropine (b) in $0.5 \mathrm{M} \mathrm{H}_{2} \mathrm{SO}_{4}$. 